\title{
Assessment of Micronutrient Status of Electronic Waste (E- waste) Recyclers at Agbogbloshie (Ghana) Using Dietary Information and Biomarker Data
}

\section{Sylvia Akpene Takyi ( $\square$ satakyi002@st.ug.edu.gh )}

Department of Biol. Env'tal \& Occupational Health Sciences School of Public Health, CHS P. O. Box LG13 University of Ghana Legon, Accra https://orcid.org/0000-0002-0211-6862

Niladri Basu

McGill University Department of Natural Resource Sciences

John Arko-Mensah

University of Ghana School of Public Health

\section{Duah Dwomoh}

University of Ghana School of Public Health

\author{
Afua Asabea Amoabeng Nti \\ University of Ghana School of Public Health \\ Lawrencia Kwarteng \\ University of Ghana School of Public Health \\ Augustine A. Acquah \\ University of Ghana School of Public Health \\ Thomas Robins \\ University of Michigan \\ Julius N. Fobil \\ University of Ghana School of Public Health
}

\section{Research}

Keywords: E-waste recyclers, dietary micronutrient intake, calcium, iron, selenium, copper, zinc, magnesium, blood, urine

Posted Date: September 8th, 2020

DOI: https://doi.org/10.21203/rs.3.rs-70898/v1

License: (c) (i) This work is licensed under a Creative Commons Attribution 4.0 International License. Read Full License 


\section{Abstract}

Background: Growing evidence suggests that heavy metals (e.g., cadmium, lead and arsenic) negatively influence micronutrient status. Electronic waste (e-waste) recyclers are amongst the highest metals-exposed groups worldwide, though their micronutrient status is yet to be explored. This study therefore assessed the micronutrient status of e-waste recyclers using dietary information and biomarker data.

Methods: Micronutrient status of 151 participants (100 e-waste recyclers and 51 controls from the Accra region, Ghana) was assessed in March 2017 using a 2-day 24-hour recall survey and biomarker (blood and urine) levels. Blood and urine levels of iron [Fe], calcium [Ca], magnesium [Mg], selenium [Se], zinc [Zn] and copper [Cu] were analyzed by ICP-MS. Linear regression models were used to assess associations between work-related factors and sociodemographic characteristics with micronutrient intake, blood and urine micronutrient levels.

Results: Dietary Fe and Zn were adequately consumed among the e-waste recyclers. Meanwhile, micronutrients-such as Ca, Se, $\mathrm{Mg}$ and $\mathrm{Cu}$-were inadequately consumed by e-waste recyclers and controls. Except for the low levels of $\mathrm{Mg}$ and Fe detected in blood of e-waste recyclers, all other micronutrients measured in both blood and urine of both groups fell within their reference range. Exposure to biomass burning was associated with lower blood levels of $\mathrm{Fe}, \mathrm{Mg}$ and $\mathrm{Zn}$ among the e-waste recyclers. Further, among e-waste recyclers, significant relationships were found between the number of years of spent recycling e-waste and urinary $\mathrm{Ca}$ and $\mathrm{Cu}$ excretion.

Conclusion: Apart from Fe and Zn, e-waste recyclers at Agbogbloshie did not meet the day to day dietary requirements for $\mathrm{Ca}$, $\mathrm{Cu}$, $\mathrm{Se}$, and $\mathrm{Mg}$ intake. In addition, exposure to biomass burning could lead to reductions in Fe, Mg, and Zn uptake in blood. Given that, blood levels of micronutrients such as $\mathrm{Mg}$ and Fe were below their reference ranges, the implementation of evidence-based nutrition strategies remains necessary among e-waste recyclers to reduce their risk of becoming malnourished.

\section{Trial Registration:}

Not applicable

\section{Background}

Adequate consumption of foods rich in micronutrients such as calcium (Ca), magnesium (Mg), iron (Fe), copper (Cu), zinc ( $\mathrm{Zn}$ ) and selenium (Se) is essential for normal physiological functions (Risk Factors Collaborators, 2016). Deficiencies in such micronutrients, perhaps owing to poor food intake, could precipitate adverse health conditions such as anaemia, hypertension, diabetes and other physiological impairments. Further, exposure to toxic metals such as cadmium (Cd), arsenic (As), and lead $(\mathrm{Pb})$ may promote micronutrient deficiency, and in turn this deficiency may aggravate the toxicity of metals. While some nutritional deficiencies may not be acutely life-threatening, they may increase one's susceptibility to chronic diseases. Given that both micronutrient deficiencies (Risk Factors Collaborators, 2016) and toxic chemical exposures (Landrigan et al., 2018) are major contributors to the global burden of disease, investigating their interactions particularly in vulnerable groups is of utmost importance.

The recycling of electronic waste (e-waste) is growing worldwide (Heacock et al., 2016; Heacock et al., 2018). It is largely an informal sector activity situated in low- and middle-income countries thus drawing from populations who likely experience micronutrient deficiencies (Basu et al., 2016). The job is physically demanding; thus, e-waste recyclers need adequate nutrient intake. Many studies have established that these recyclers are exposed to many toxic chemicals (Cao et al., 2019; Singh et al., 2018; Srigboh et al., 2016; Wittsiepe et al., 2017). The interactions between micronutrient and toxic chemicals including heavy metals are not fully known, albeit these are likely to impact on each other. For instance, toxic metals and absorbed micronutrients may interact at several points in the body (i.e., during absorption, excretion, transport or binding to target proteins). Micronutrient deficiencies may potentiate the toxicity of hazardous metals in the body (Jan et al., 2015; Schwalfenberg \& Genuis, 2015). Consistent with this observation, adequate nutrition also serves as an essential factor in preventing toxic metal poisoning (Kordas et al., 2017), as poor nutrition status impairs the body's defence system against exposure to toxic metals (Tchounwou et al., 2012). Several studies have investigated metals exposure among e-waste recyclers (Cao et al., 2019; Singh et al., 2018; Srigboh et 
al., 2016; Wittsiepe et al., 2017). However, no attention has been given to investigating the micronutrient status of e-waste recyclers, despite the fact that they are continuously exposed to toxic metals and other environmental contaminants believed to negatively affect nutritional status (Erickson \& Arbour, 2014; Hennig et al., 2007; Kannan et al., 2006; Miller \& Rayalam, 2017).

In Ghana, the primary e-waste site is Agbogbloshie situated in central Accra (Basu et al., 2016) with several thousand recyclers dependent upon the industry (Prakash et al., 2010). Past studies have characterized relatively high exposures of recyclers at Agbogbloshie to a diverse range of chemicals (Srigboh et al., 2016; Wittsiepe et al., 2017), yet to our knowledge, no assessment of their micronutrient status has been done. We, therefore, addressed this critical knowledge gap by conducting a cross-sectional study at Agbogbloshie to (i) assess micronutrient ( $\mathrm{Ca}, \mathrm{Mg}, \mathrm{Se}, \mathrm{Fe}, \mathrm{Zn}$ and $\mathrm{Cu}$ ) intake (based on dietary surveys), (ii) measure the levels of these micronutrients in the blood and urine, (iii) assess the relationship between dietary micronutrient intake and the micronutrient levels in blood and urine, and (iv) examine the associations between work-related factors and sociodemographic characteristics on dietary micronutrient intake, as well as the micronutrient levels in the blood and urine of the e-waste recyclers and controls.

\section{Methods}

\section{Study Design}

\section{Data Source and Study Design}

Our study data were drawn from the GEOHealth-II longitudinal cohort study (Amoabeng Nti et al., 2020; Kwarteng et al., 2020; Laskaris et al., 2019; Takyi et al., 2020). We carried out a cross-sectional study of male e-waste recyclers ( $N=100$ from the Agbogbloshie site) and controls ( $\mathrm{N}=51$ controls from Madina, a community about $10 \mathrm{~km}$ south of Agbogbloshie), all in Accra in March 2017. As detailed in our previous studies at Agbogbloshie (Asampong et al., 2015; Feldt et al., 2014; Srigboh et al., 2016; Wittsiepe et al., 2017), initially we organized a community durbar to inform, and also familiarize, eligible participants with the study's objectives and procedures. The inclusion criteria were adult males aged 18 years and above who had worked at the ewaste site for at least six months. Similarly, participants from the control site (Madina-Zongo, Accra, Ghana) were in the same age category and with similar demographic characteristics as the e-waste recyclers (e.g., culture and dietary habits). In addition, participants from the control site must have lived there for at least six months. Informed consent was obtained from all participants, and they were compensated with 50 Ghanaian cedis (approximately \$10 USD, which is roughly an average day's wage), a lunch pack and T-shirt. Institutional Review Boards at the University of Ghana, the University of Michigan and McGill University approved the study protocols. Also, the local chief of Agbogbloshie and Madina-Zongo permitted our research team to enter the community to conduct this study.

\section{Study Site}

Agbogbloshie is an informal e-waste dumping area, scrap processing and food market zone located on the bank of the KorleLagoon, to the west of the Odaw River in the heart of Accra. The site is geographically hemmed within the areas of Abossey Okai road, the Odaw river and the Agbogbloshie drain. Nearly all the e-waste recyclers are Muslims from either the Dagomba or Konkomba ethnic groups, who migrated from the northern part of Ghana in search of improved employment opportunities. The Odaw river marks the border of the e-waste site and Old Fadama slum, which houses about 80,000 people (Simon, 2018; United Nations Population Fund, 2018).

This study also included a control group residing in Madina-Zongo who have similar socio-demographic characteristics with the e-waste recyclers with respect to religion and internal migration from northern parts of Ghana. Madina-Zongo is located within 10 $\mathrm{km}$ of the Agbogbloshie e-waste site and is expected to be unexposed to e-waste activities.

\section{Field Data Collection Procedures}

\section{Anthropometric Measurements}


The participant's height and weight were measured using a standardized protocol. Height was measured with a Seca stadiometer (Seca, Hamburg, Germany), and corrected to the nearest $0.1 \mathrm{~cm}$, with participant standing upright on a flat surface without shoes, and the back of the heels and the occiput against the stadiometer (Alkhajah et al., 2012; Boateng, 2014; Zeba et al., 2014). Also, participant's body weight was measured to the nearest $0.1 \mathrm{~kg}$ using a portable Seca scale (Seca 770, Hamburg, Germany). The same model of a standard calibrated balance was used at both study sites. The body mass index (BMI) of the participants was calculated by dividing their weight in kilograms $(\mathrm{kg})$ by height in meters squared $\left(\mathrm{m}^{2}\right)$.

Based on previous exposure assessments and occupational health surveys conducted in Ghana (Nti, 2015; Srigboh et al., 2016; Wittsiepe et al., 2017), a semi-structured questionnaire was designedpiloted and used to collect information on the sociodemographic characteristics and work history of the participants.

\section{Dietary Micronutrient Intake Assessment}

Data collection took place over a period of two weeks in each study site. Daily nutritional intake of the participants was collected using a semi-structured 2-day 24-hour recall guide. We conducted the 24-hour recall twice to estimate the day-to-day variability that occurs per individual owing to the variety of foods consumed on different days. In an effort to maximize the consistency of the interview format across the study sites and also minimize between site methodological biases, trained dieticians were used as interviewers. The interview was conducted in local dialects of the participants' native or preferred language-Dagbani, Hausa, Twi or English-to ensure that the participants fully understood the questions asked with respect to the 2-day 24-hour recall guide. The interview consisted of questions on foods and beverages (e.g., the amount, the time and the types of meals/foods) consumed on one weekday and one day of preceding weekend (Saturday or Sunday). In all cases, information was solicited within less than 24 hours when that day ended. This was done either face to face or through phone calls. We also used graduated food models (FAO, 2018; Hansen et al., 2011) to quantify foods and beverages consumed by each participant.

\section{Blood and Urine Sample Collection}

Urine and whole blood samples were collected in a clean and enclosed area near each of the study sites. A midstream urine sample $(15 \mathrm{ml})$ was collected into a plastic container at the start of each participant's visit to the clinic, typically between the hours of 9 a.m. and 4 p.m. Approximately $10 \mathrm{ml}$ of blood was collected into a trace metal-free BD Vacutainer tube with $\mathrm{K}_{2}$ EDTA, and placed on a blood tube roller (Micro-Teknik) for five minutes. The urine and blood samples were stored in a $-80^{\circ} \mathrm{C}$ freezer until shipment to McGill University (Montreal, Canada) where they were stored frozen $\left(-80^{\circ} \mathrm{C}\right)$ prior to analyses.

\section{Data Analysis}

\section{Dietary Micronutrient Intake Analysis}

The dietary micronutrient intake data collected was converted into grams using Ghanaian food composition tables. We further undertook a comprehensive nutrient analysis using the ESHA F Pro ${ }^{\circledR}$ software to estimate individual micronutrient intake. Data obtained from the ESHA F Pro after nutrient analysis consisted of $\mathrm{Ca}, \mathrm{Mg}, \mathrm{Fe}, \mathrm{Zn}, \mathrm{Cu}$ and $\mathrm{Se}$. These results were compared to the recommended daily allowance (RDA) for adult males (Mahan \& Raymond, 2016).

\section{Laboratory Analysis of Micronutrients in the Blood and Urine}

Micronutrients-namely, $\mathrm{Ca}, \mathrm{Mg}, \mathrm{Fe}, \mathrm{Se}, \mathrm{Cu}$ and $\mathrm{Zn}$-were analyzed in both whole blood and urine. The urine and blood samples were acid-digested as outlined in previous studies (Basu et al., 2011; Srigboh et al., 2016). Elemental concentrations were determined using an inductively coupled plasma mass spectrometer (ICPMS; Varian 820MS). Several analytical quality control measures were used. All tubes and pipette tips used were acid-washed (cleaned, soaked for $24 \mathrm{hrs}$ in $10 \%$ hydrochloric acid and rinsed three times in Milli-Q water) prior to usage. Certified standard reference materials (INSPQ; QM-U-Q1109 [urine]; and then QM-B-Q1506 and QM-B-Q1314 [blood]) obtained from the Institut National de Santé Publique du Québec were used to measure accuracy and precision. Furthermore, each batch run consisted of procedural blanks and replicates. For each micronutrient analyzed, the theoretical detection limit $(\mu \mathrm{g} / \mathrm{L})$ was calculated as three times the standard deviation of the mean blank value (Supplemental Table 1). 


\section{Statistical Analysis}

Dietary micronutrient intake ( $\mathrm{Ca}, \mathrm{Mg}, \mathrm{Fe}, \mathrm{Zn}, \mathrm{Cu}$ and Se), participant work status (e-waste recycler vs control), job task (dismantlers, burners and collectors/sorters), work characteristics (e.g., daily duration (hours) of e-waste work activity and number of years spent recycling e-waste), and other sociodemographic characteristics (e.g., educational status, daily income earned and age) were our primary exposures of interest. Environmental risk factors such as cigarette smoking, exposure to biomass burning and alcohol intake were also notable exposures of interest. Depending on the model investigated, outcome variables were either micronutrient levels ( $\mathrm{Ca}, \mathrm{Mg}, \mathrm{Fe}, \mathrm{Zn}, \mathrm{Cu}$ and $\mathrm{Se}$ ) in blood and urine or dietary micronutrient intake values.

Data analysis focused on the following a priori questions. First, to characterize dietary micronutrient intake, the fraction of ewaste recyclers and the controls who consumed adequate amounts of micronutrients was assessed by defining and dichotomizing each of the micronutrients based on the United States Department of Agriculture (USDA) guidelines (Moore et al., 2012) for adults. This definition outlines the threshold for micronutrient adequacy using data obtained from the micronutrient intake. The Kruskal-Wallis test was used to determine whether differences existed between the amounts of micronutrients consumed from diet by the e-waste recyclers and the controls. We further used the Wilcoxon test to assess the differences in mean dietary micronutrient levels among the e-waste recycler groups (burners, dismantlers and collectors/sorters). Second, to compare the levels of micronutrients in the whole blood and urine of the e-waste recyclers and the controls, the Kruskal-Wallis test was used. The Wilcoxon test was further used to examine the differences in biomarker micronutrient levels between the e-waste recycler groups. Third, relationships between dietary micronutrient intakes and micronutrient levels in blood and urine were gauged using Pearson's correlation. Fourth, to increase understanding of the micronutrient data (both dietary and biomarkerbased), they were associated with associated with sociodemographic characteristics and work-related factors through regression modelling. Outliers were identified as values that were more than three standard deviations away from the mean, and these were removed from the dataset. Stata ${ }^{\circledR}$ version 15 (StataCorp, College Station, Texas, USA) was used for data analysis.

\section{Results}

\section{Sociodemographic Characteristics of the E-waste Recyclers and Controls}

The mean \pm SD age of the e-waste recyclers and the controls assessed was $25.4 \pm 6.3$ and $32.3 \pm 10.2$ years, respectively. BMI was significantly higher in the controls $\left(23.9 \mathrm{~kg} / \mathrm{m}^{2}\right)$ than e-waste recyclers $\left(21.8 \mathrm{~kg} / \mathrm{m}^{2}\right)$ but was within a healthy body weight range for both groups (Supplementary Table 2). Majority of the e-waste recyclers (92\%) and controls (84.3\%) were Muslims (Table 1). More than half $(55 \%)$ of e-waste recyclers were married, compared to $(58.8 \%)$ of the controls who were single. Controls have had better education than e-waste recyclers; $41.3 \%$ of controls had completed Senior Secondary School, compared to just a third of the e-waste recyclers (32.3\%) who reported to have completed Junior High School. Two thirds (63.6\%) of e-waste recyclers earned between 20 and 100 Ghanaian cedis daily, and a $24.2 \%$ earned less than 20 Ghanaian cedis per day ( \$4); and only $4 \%$ earned more than 200 Ghanaian cedis $(\sim 35)$ per day. Furthermore, daily income earned was neither associated with educational status $\left(x^{2}=4.42, p=0.98\right)$ nor job task $\left(x^{2}=6.73, p=0.35\right)$ undertaken at the e-waste recycling site. More than half of the e-waste recyclers (55.2\%) slept on-site, while the rest lived off-site either within $1 \mathrm{~km}$ of Agbogbloshie (36.5\%) or more than $1 \mathrm{~km}$ away (8.3\%). The recyclers reported to work approximately 9 hours per day for about 10 years. Finally, $36 \%$ of the e-waste recyclers were highly exposed to biomass burning than the controls $(29 \%)$.

\section{Table 1: Socio-demographic characteristics of e-waste recyclers and control group.}




\begin{tabular}{|c|c|c|c|c|c|}
\hline Demographics & $\begin{array}{l}\text { Total } \\
\mathbf{N}\end{array}$ & $\begin{array}{l}\text { E-waste recyclers } \\
\text { n (\%) }\end{array}$ & $\begin{array}{l}\text { Controls } \\
n(\%)\end{array}$ & $x^{2}$ & $p$-value \\
\hline Marital Status & 150 & & & 4.43 & 0.06 \\
\hline Single & & $44(44.4)$ & $31(60.8)$ & & \\
\hline Married & & $55(55.6)$ & $20(39.2)$ & & \\
\hline Daily Income & 149 & & & 5.12 & 0.16 \\
\hline$\leq$ GHS 20 & & $24(24.2)$ & $9(18.0)$ & & \\
\hline GHS 21-100 & & $63(63.6)$ & $30(60.0)$ & & \\
\hline GHS 101-200 & & $8(8.1)$ & $4(8.0)$ & & \\
\hline GHS >200 & & $4(4.0)$ & $7(14.0)$ & & \\
\hline Education & 145 & & & 23.82 & $<0.01$ \\
\hline None & & $25(25.2)$ & $6(13.0)$ & & \\
\hline Primary & & $26(26.3)$ & $4(8.7)$ & & \\
\hline Middle/JSS & & 32 (32.3) & $12(26.1)$ & & \\
\hline Secondary/SSS \& Higher & & $16(16.2)$ & $24(52.3)$ & & \\
\hline Religion & 150 & & & 3.45 & 0.18 \\
\hline Muslim & & 92 (92.9) & 43 (84.3) & & \\
\hline Christian & & $5(5.1)$ & $7(13.7)$ & & \\
\hline Others & & $2(2)$ & $1(2)$ & & \\
\hline Smoking & 146 & $27(27.8)$ & $6(12.4)$ & 4.52 & 0.03 \\
\hline Alcohol intake & 151 & $17(17.0)$ & $9(17.7)$ & 0.01 & 0.92 \\
\hline E-waste Job-task & 100 & & & NA & NA \\
\hline Burners & & $32(32)$ & NA & & \\
\hline Dismantlers & & $49(49)$ & NA & & \\
\hline Collectors/ Sorters & & 19 (19) & NA & & \\
\hline
\end{tabular}

\section{Abbreviation: SD; Standard Deviation; NA: Not applicable}

\section{Dietary micronutrient intake (based on food models) of the -waste recyclers and the controls.}

Dietary micronutrient intake is summarized in Table 2a. The dietary intake of Fe and $\mathrm{Zn}$ was significantly higher among the ewaste recyclers than controls, whereas $\mathrm{Mg}$ intake was higher among the controls (Table 2a). For other micronutrients (Ca, Se, Fe, $\mathrm{Mg}, \mathrm{Zn}, \mathrm{Cu}$ ) assessed, there were no differences in consumption between e-waste recyclers and controls. For e-waste recyclers, the mean dietary intake of $\mathrm{Ca}, \mathrm{Se}, \mathrm{Mg}, \mathrm{Zn}$, and $\mathrm{Cu}$ was highest among those who were primarily involved in dismantling e-waste. In contrast, recyclers who were primarily involved in open-air burning of e-waste to retrieve valuable parts, especially Cu did not consume diets with adequate amounts of $\mathrm{Ca}, \mathrm{Zn}, \mathrm{Cu}$ and Fe. Overall, nearly all the e-waste recyclers (96\%) and the controls (98\%) met the RDA for Fe in this study. However, only a few of the e-waste recyclers ( $<30 \%)$ met the RDA for Ca, Mg, Se, $\mathrm{Zn}$ and $\mathrm{Cu}$ (Supplementary Table).

Table 2a: Dietary micronutrient intake (based on food models) of e-waste recyclers and controls. 


\begin{tabular}{|lllllll|}
\hline Dietary Micronutrient & \multicolumn{5}{l}{ E-waste Recyclers } & $\underline{\text { Controls }}$ \\
\hline intake $(\mathrm{mg})$ & RDA $(\mathrm{mg})$ & Mean \pm SD & Median (IQR) & Mean \pm SD & Median (IQR) & $p$-value \\
\hline $\mathrm{Ca}$ & 1000 & $534.4 \pm 352.3$ & $449(375.1)$ & $557.0 \pm 369.6$ & $497(321)$ & 0.47 \\
$\mathrm{Mg}$ & 350 & $45.7 \pm 44.2$ & $33.3(38.1)$ & $84.7 \pm 67.3$ & $59.6(86.8)$ & $<0.01$ \\
$\mathrm{Se}$ & 55 & $22.8 \pm 18.0$ & $17.9(20.4)$ & $32.2 \pm 39.9$ & $19.8(36.2)$ & 0.50 \\
$\mathrm{Zn}$ & 11 & $11.3 \pm 4.1$ & $10.6(4.6)$ & $9.7 \pm 4.7$ & $8.7(5.9)$ & 0.01 \\
$\mathrm{Cu}$ & 2 & $1.1 \pm 0.6$ & $1.0(0.6)$ & $1.0 \pm 0.7$ & $1.1(0.6)$ & 0.12 \\
\hline $\mathrm{Fe}$ & 8 & $26.5 \pm 13.0$ & $24.2(15.9)$ & $22.4 \pm 10.5$ & $24.8(14.4)$ & 0.04 \\
\hline
\end{tabular}

Abbreviations: RDA-Recommended Daily Intake; SD: Standard deviation; IQR: Interquartile range. P-value estimate from the Wilcoxon rank sum test

Table 2b: Dietary micronutrient intake (based on food models) of key e-waste recycler groups. The groups were determined based on self-reported data, though recycler tend to perform a variety of tasks.

\begin{tabular}{|c|c|c|c|c|c|c|c|c|}
\hline \multirow{2}{*}{$\begin{array}{l}\text { Dietary Micronutrient } \\
\text { intake }(\mathrm{mg})\end{array}$} & \multirow{2}{*}{$\begin{array}{l}\text { RDA } \\
(\mathrm{mg})\end{array}$} & \multicolumn{2}{|l|}{ Burner } & \multicolumn{2}{|l|}{ Dismantler } & \multicolumn{2}{|c|}{ Collector/Sorter } & \multirow{2}{*}{$\begin{array}{l}p- \\
\text { value }\end{array}$} \\
\hline & & Mean $\pm S D$ & $\begin{array}{l}\text { Median } \\
\text { (IQR) }\end{array}$ & Mean $\pm S D$ & $\begin{array}{l}\text { Median } \\
\text { (IQR) }\end{array}$ & Mean $\pm S D$ & $\begin{array}{l}\text { Median } \\
\text { (IQR) }\end{array}$ & \\
\hline $\mathrm{Ca}$ & 1000 & $453.3 \pm 164.8$ & $\begin{array}{l}418.3 \\
(202.5)\end{array}$ & $582.8 \pm 439.1$ & $\begin{array}{l}468.5 \\
(400.6)\end{array}$ & $546.3 \pm 321.5$ & $\begin{array}{l}534.5 \\
(376)\end{array}$ & 0.67 \\
\hline $\mathrm{Mg}$ & 350 & $46.1 \pm 38.4$ & $\begin{array}{l}39.9 \\
(50.6)\end{array}$ & $49.7 \pm 52.5$ & $\begin{array}{l}34.3 \\
(32.7)\end{array}$ & $34.1 \pm 24.7$ & $\begin{array}{l}26.9 \\
(38.3)\end{array}$ & 0.48 \\
\hline $\mathrm{Se}$ & 55 & $21.8 \pm 18.3$ & $\begin{array}{l}16.3 \\
(21.4)\end{array}$ & $24.3 \pm 18.7$ & $\begin{array}{l}19.0 \\
(18.1)\end{array}$ & $20.9 \pm 16.3$ & $\begin{array}{l}16.4 \\
(24.6)\end{array}$ & 0.57 \\
\hline $\mathrm{Zn}$ & 11 & $10.5 \pm 4.2$ & $\begin{array}{l}10.3 \\
(5.7)\end{array}$ & $11.9 \pm 4.3$ & $\begin{array}{l}11.4 \\
(5.1)\end{array}$ & $11.2 \pm 3.3$ & $\begin{array}{l}10.6 \\
(4.3)\end{array}$ & 0.61 \\
\hline $\mathrm{Cu}$ & 2 & $1.0 \pm 0.5$ & $1.0(0.7)$ & $1.2 \pm 0.7$ & $1.1(0.6)$ & $1.1 \pm 0.5$ & $\begin{array}{l}1.0 \\
(0.8)\end{array}$ & 0.79 \\
\hline $\mathrm{Fe}$ & 8 & $23.9 \pm 12.9$ & $\begin{array}{l}22.7 \\
(13.3)\end{array}$ & $27.7 \pm 13.1$ & $\begin{array}{l}24.8 \\
(14.4)\end{array}$ & $27.8 \pm 12.9$ & $\begin{array}{l}25.4 \\
(18.6)\end{array}$ & 0.35 \\
\hline
\end{tabular}

Abbreviations; SD: standard deviation; IQR: interquartile range; Ca: Calcium; Mg: Magnesium; Fe: Iron; Se: Selenium; Cu: Copper, Zn: Zinc. p-value estimate from the Kruskal wallis test

\section{Micronutrient Biomarkers levels (Descriptive Summary)}

In general, both the blood and urine datasets were deemed fit for analyses in terms of our review of quality control parameters. The average recovery (accuracy) of elements from the blood standard reference materials (SRM) used was $94 \%$ of the expected value for Se, Cu and Zn (Supplemental table 1). However, the mean analytical precision of all blood elements was $9 \%$. In urine, the mean recovery

(accuracy) was between $63 \%$ and $123 \%$ for $\mathrm{Cu}, \mathrm{Zn}$ and Se. Analytical precision for the urinary micronutrient measured was $15 \%$. The accuracy of $\mathrm{Ca}$, Fe and $\mathrm{Mg}$ in blood and urine could not be calculated as the reference materials used did not include guidance values for these. 
For whole blood and urine samples analyzed, the mean concentrations obtained for $\mathrm{Ca}, \mathrm{Mg}, \mathrm{Fe}, \mathrm{Zn}$, Se, and Cu among the e-waste recyclers and the controls are reported in Table 3a. We further tabulated these biomarker values per job tasks performed (Table 3b).

Due to the unavailability of suitable biomarker reference levels for Ghana (or Africa), the mean micronutrient levels of $\mathrm{Fe}, \mathrm{Zn}, \mathrm{Cu}$ and Se in blood and urine were compared to values proposed by lyengar and Woittiez (1988) in their effort to establish "normal values" for elements in biological samples. However, blood and urinary levels of $\mathrm{Ca}$ and $\mathrm{Mg}$ were compared to reference ranges established by Alimonti et al. (2005). In all, median levels of $\mathrm{Ca}$, Zn and Se measured in whole blood of both e-waste recyclers and controls fell within these reference ranges. To ensure consistency, we also compared the urinary micronutrient levels with reference ranges established by lyengar et al. (1988) and Alimonti et al. (2005). The estimated levels of the micronutrients in urine were within the normal range in both the e-waste recyclers and controls.

The median blood levels of $\mathrm{Mg}$, Se, and Fe were significantly lower among the e-waste recyclers than controls $(p<0.05)$. Furthermore, among the e-waste recycler groups, median levels of $\mathrm{Ca}, \mathrm{Mg}, \mathrm{Zn}$, and Se in blood were considerably highest among those who self-reported that they primarily collect/sort e-waste, followed by the dismantlers and then the burners. Significant differences were found in urinary levels of $\mathrm{Ca}$, Se, and Fe among the e-waste recyclers and controls $(p<0.01)$. While urinary $\mathrm{Ca}$ and Se levels were higher among the controls, increased amounts of Cu were excreted among the e-waste recyclers $(p<0.01)$. Urinary $\mathrm{Zn}$ and $\mathrm{Cu}$ levels differed among the e-waste recycler groups $(p<0.01)$. While $\mathrm{Zn}$ was particularly high in urine of the collectors/ sorters, Cu was highly removed in urine of the burners.

Table 3a: Micronutrient levels in blood and urine of e-waste recyclers and controls

\begin{tabular}{|c|c|c|c|c|c|c|}
\hline \multirow{2}{*}{$\begin{array}{l}\text { Micronutrient Levels } \\
(\mu \mathrm{g} / \mathrm{L})\end{array}$} & \multirow{2}{*}{$\begin{array}{l}\text { Reference } \\
\text { range }\end{array}$} & \multicolumn{2}{|l|}{ E-waste recyclers } & \multicolumn{2}{|l|}{ Controls } & \multirow{2}{*}{$\begin{array}{l}p- \\
\text { value }\end{array}$} \\
\hline & & Mean $\pm S D$ & Median (IQR) & Mean $\pm S D$ & Median (IQR) & \\
\hline \multicolumn{7}{|l|}{ Whole Blood } \\
\hline $\mathrm{Ca}$ & $\begin{array}{l}59028- \\
72193\end{array}$ & $\begin{array}{l}61992.1 \pm \\
13610.6\end{array}$ & $\begin{array}{l}63155.9 \\
(16083.2)\end{array}$ & $\begin{array}{l}65851.8 \pm \\
9270.3\end{array}$ & $\begin{array}{l}65629.4 \\
(8835.7)\end{array}$ & 0.07 \\
\hline $\mathrm{Mg}$ & $\begin{array}{l}36951- \\
43276\end{array}$ & $\begin{array}{l}33717.2 \pm \\
5694.1\end{array}$ & $\begin{array}{l}34581.0 \\
(6703.3)\end{array}$ & $\begin{array}{l}38363.8 \pm \\
4653.1\end{array}$ & $\begin{array}{l}38560.7 \\
(6481.2)\end{array}$ & $<0.01$ \\
\hline Se & $58-234$ & $152.9 \pm 39.8$ & $145.9(56.9)$ & $194.9 \pm 44.1$ & 193.1 (49.4) & $<0.01$ \\
\hline $\mathrm{Zn}$ & $4837-7980$ & $\begin{array}{l}7879.3 \pm \\
2782.3\end{array}$ & $7242.9(3267.6)$ & $\begin{array}{l}8355.2 \pm \\
2466.9\end{array}$ & $8057.9(2043.2)$ & 0.09 \\
\hline $\mathrm{Cu}$ & $683-1036$ & $1107.9 \pm 222.6$ & 1110.9 (233.5) & $1143.0 \pm 234.1$ & $1107.7(259.6)$ & 0.72 \\
\hline $\mathrm{Fe}$ & $\begin{array}{l}390000- \\
550000\end{array}$ & $\begin{array}{l}370697.2 \pm \\
68181.2\end{array}$ & $\begin{array}{l}381307.2 \\
(77495.4)\end{array}$ & $\begin{array}{l}404169.7 \pm \\
65185.7\end{array}$ & $\begin{array}{l}427274.6 \\
(88040.3)\end{array}$ & $<0.01$ \\
\hline \multicolumn{7}{|l|}{ Urine } \\
\hline $\mathrm{Ca}$ & $\begin{array}{l}67000- \\
200000\end{array}$ & $\begin{array}{l}66419.2 \pm \\
75335.6\end{array}$ & $\begin{array}{l}40243.9 \\
(62094.9)\end{array}$ & $\begin{array}{l}90814.3 \pm \\
69028.3\end{array}$ & $\begin{array}{l}71103.2 \\
(96152.5)\end{array}$ & $<0.01$ \\
\hline $\mathrm{Mg}$ & $\begin{array}{l}15000- \\
120000\end{array}$ & $\begin{array}{l}82220.5 \pm \\
55215\end{array}$ & $\begin{array}{l}71291.8 \\
(72978.9)\end{array}$ & $\begin{array}{l}82000.5 \pm \\
56931.5\end{array}$ & $\begin{array}{l}76954.6 \\
(63733.0)\end{array}$ & 0.98 \\
\hline $\mathrm{Se}$ & $7-160$ & $29.7 \pm 18.8$ & $26.7(22.3)$ & $44.5 \pm 25.8$ & $39.9(31.1)$ & $<0.01$ \\
\hline $\mathrm{Zn}$ & $700-2500$ & $\begin{array}{l}3718.7 \pm \\
7942.6\end{array}$ & $1044.4(5022.0)$ & $\begin{array}{l}1561.8 \pm \\
1294.2\end{array}$ & $1253.0(1195.7)$ & 0.89 \\
\hline $\mathrm{Cu}$ & $12-80$ & $63.7 \pm 53.8$ & $38.1(76.6)$ & $110.5 \pm 567.8$ & $25.6(16.0)$ & $<0.01$ \\
\hline $\mathrm{Fe}$ & $1.2-600$ & $181.4 \pm 351.8$ & $95.4(79.8)$ & $114.4 \pm 97.8$ & $85.2(52.3)$ & 0.08 \\
\hline
\end{tabular}

Abbreviations: Ca: Calcium; Mg: Magnesium; Fe: Iron; Se: Selenium; Cu: Copper; Zn: Zinc 
Sources of reference ranges: Se, Fe, Zn, Cu -lyengar and Woittiez (1988); whiles Ca and Mg were from-Alimonti et al. (2005) in blood and urine.

Table 3b: Micronutrient levels in blood and urine of e-waste recycler groups

\begin{tabular}{|c|c|c|c|c|c|c|c|c|}
\hline \multirow{2}{*}{$\begin{array}{l}\text { Micronutrient } \\
\text { levels }(\mu \mathrm{g} / \mathrm{L})\end{array}$} & \multirow{2}{*}{$\begin{array}{l}\text { Reference } \\
\text { range }\end{array}$} & \multicolumn{2}{|l|}{ Burner $(\mathrm{n=32})$. } & \multicolumn{2}{|c|}{ Dismantler $(\underline{n=49})$. } & \multicolumn{2}{|c|}{ Collector/Sorter $(\underline{n=19})$. } & \multirow{2}{*}{$\begin{array}{l}p- \\
\text { value }\end{array}$} \\
\hline & & Mean $\pm S D$ & $\begin{array}{l}\text { Median } \\
\text { (IQR) }\end{array}$ & Mean $\pm S D$ & $\begin{array}{l}\text { Median } \\
\text { (IQR) }\end{array}$ & Mean $\pm S D$ & $\begin{array}{l}\text { Median } \\
\text { (IQR) }\end{array}$ & \\
\hline \multicolumn{9}{|l|}{ Whole Blood } \\
\hline $\mathrm{Ca}$ & $\begin{array}{l}59028- \\
72,193\end{array}$ & $55577.0 \pm 13646.0$ & $\begin{array}{l}55429.0 \\
(18578.1)\end{array}$ & $\begin{array}{l}62995.2 \\
\pm \\
11426.7\end{array}$ & $\begin{array}{l}63719.6 \\
(12923.5)\end{array}$ & $\begin{array}{l}70209.5 \\
\pm \\
14240.9\end{array}$ & $\begin{array}{l}68755.8 \\
(11117.3)\end{array}$ & $<0.01$ \\
\hline $\mathrm{Mg}$ & $\begin{array}{l}36951- \\
43,276\end{array}$ & $30991.6 \pm 6462.2$ & $\begin{array}{l}32633.0 \\
(7851.5)\end{array}$ & $\begin{array}{l}34903.6 \\
\pm 5403.7\end{array}$ & $\begin{array}{l}35413.4 \\
(6951.0)\end{array}$ & $\begin{array}{l}35248.3 \\
\pm 5132.2\end{array}$ & $\begin{array}{l}36003.3 \\
(6693.6)\end{array}$ & 0.02 \\
\hline Se & $58-234$ & $135.1 \pm 34.1$ & $\begin{array}{l}129.5 \\
(33.8)\end{array}$ & $\begin{array}{l}158.5 \pm \\
41.8\end{array}$ & $\begin{array}{l}159.7 \\
(63.3)\end{array}$ & $\begin{array}{l}168.6 \pm \\
33.9\end{array}$ & $\begin{array}{l}162.4 \\
(51.5)\end{array}$ & $<0.01$ \\
\hline Zn & $\begin{array}{l}4837- \\
7980\end{array}$ & $6496.3 \pm 2114.9$ & $\begin{array}{l}6667.9 \\
(2356.9)\end{array}$ & $\begin{array}{l}8105.2 \pm \\
2594.0\end{array}$ & $\begin{array}{l}7571.8 \\
(2747.2)\end{array}$ & $\begin{array}{l}9626.1 \pm \\
3189.2\end{array}$ & $\begin{array}{l}10069 \\
(4126.8)\end{array}$ & $<0.01$ \\
\hline $\mathrm{Cu}$ & 683-1036 & $1038.4 \pm 249.3$ & $\begin{array}{l}1028.6 \\
(324.4)\end{array}$ & $\begin{array}{l}1126.5 \pm \\
218.5\end{array}$ & $\begin{array}{l}1107.8 \\
(217.7)\end{array}$ & $\begin{array}{l}1173.6 \\
\pm 155.2\end{array}$ & $\begin{array}{l}1131.6 \\
(198.1)\end{array}$ & 0.12 \\
\hline $\mathrm{Fe}$ & $\begin{array}{l}390000- \\
550000\end{array}$ & $350216.2 \pm 78098.1$ & $\begin{array}{l}368696.2 \\
(120304.8)\end{array}$ & $\begin{array}{l}382397.1 \\
\pm \\
58722.5\end{array}$ & $\begin{array}{l}384963.9 \\
(67423.7)\end{array}$ & $\begin{array}{l}375166.5 \\
\pm \\
68887.8\end{array}$ & $\begin{array}{l}389211.4 \\
(75233.2)\end{array}$ & 0.19 \\
\hline \multicolumn{9}{|l|}{ Urine } \\
\hline $\mathrm{Ca}$ & $\begin{array}{l}67000- \\
200000\end{array}$ & $61621.6 \pm 73714.8$ & $\begin{array}{l}34674.6 \\
(67557.2)\end{array}$ & $\begin{array}{l}58091.7 \\
\pm \\
47212.3\end{array}$ & $\begin{array}{l}53992.3 \\
(60345.3)\end{array}$ & $\begin{array}{l}95099.1 \\
\pm \\
120164.4\end{array}$ & $\begin{array}{l}40548.7 \\
(79110.5)\end{array}$ & 0.44 \\
\hline Mg & $\begin{array}{l}15000- \\
120000\end{array}$ & $85002.8 \pm 62528.2$ & $\begin{array}{l}66585.8 \\
(81500.2)\end{array}$ & $\begin{array}{l}78503.6 \\
\pm \\
53177.6\end{array}$ & $\begin{array}{l}71412.0 \\
(67174.9)\end{array}$ & $\begin{array}{l}86729.0 \\
\pm \\
48965.0\end{array}$ & $\begin{array}{l}86529.7 \\
(59442.0)\end{array}$ & 0.70 \\
\hline Se & $7-160$ & $26.3 \pm 20.2$ & $20.7(19.9)$ & $\begin{array}{l}32.3 \pm \\
19.2\end{array}$ & $\begin{array}{l}27.1 \\
(22.9)\end{array}$ & $\begin{array}{l}31.6 \pm \\
15.1\end{array}$ & $\begin{array}{l}32.3 \\
(23.3)\end{array}$ & 0.15 \\
\hline $\mathrm{Zn}$ & $700-2500$ & $1548.1 \pm 2012.8$ & $\begin{array}{l}784.5 \\
(769.1)\end{array}$ & $\begin{array}{l}5368.0 \pm \\
11138.6\end{array}$ & $\begin{array}{l}1047.6 \\
(5022)\end{array}$ & $\begin{array}{l}3468.2 \pm \\
2729.4\end{array}$ & $\begin{array}{l}4597.4 \\
(5473.2)\end{array}$ & $<0.01$ \\
\hline $\mathrm{Cu}$ & $12-80$ & $87.7 \pm 57.6$ & $97.6(87.3)$ & $\begin{array}{l}48.3 \pm \\
57.6\end{array}$ & $\begin{array}{l}87.3 \\
(82.6)\end{array}$ & $\begin{array}{l}61.2 \pm \\
64.8\end{array}$ & $\begin{array}{l}33.8 \\
(43.0)\end{array}$ & $<0.01$ \\
\hline $\mathrm{Fe}$ & $1.2-600$ & $108.9 \pm 69.4$ & $87.3(82.6)$ & $\begin{array}{l}108.9 \pm \\
69.4\end{array}$ & $\begin{array}{l}100.4 \\
(68.9)\end{array}$ & $\begin{array}{l}426.5 \pm \\
744.7\end{array}$ & $\begin{array}{l}100.4 \\
(153.6)\end{array}$ & 0.19 \\
\hline
\end{tabular}

Abbreviation: SD: Standard deviation; IQR: Interquartile range; Ca: Calcium; Mg: Magnesium; Fe: Iron; Se: Selenium; Cu: Copper; $\mathrm{Zn}$ :

Sources of reference ranges: Se, Fe, Zn, Cu -lyengar and Woittiez (1988); whiles Ca and Mg were from-Alimonti et al. (2005) in blood and urine.

Relationship between dietary micronutrient intake and micronutrient levels in the blood and urine of e-waste recyclers 
The correlations between dietary micronutrient intake and levels of micronutrient in the blood and urine of e-waste recyclers are presented in Table 4. Between dietary intake values and blood levels, few of these relationships were significantly positive (5 out of 36; Mg-Fe, Ca-Zn, Mg-Zn, Mg-Se and Mg-Mg), whereas for urine only one associated was significantly positive (Mg-Cu) and $\sim 50 \%$ of them were negative.

Table 4: Correlation coefficients between dietary (D) micronutrient intake (top row) and micronutrient levels in blood (B) and urine (U) of e-waste recyclers (first column). Bolded numbers indicate correlations that are of statistical significance.

\begin{tabular}{|c|c|c|c|c|c|c|}
\hline Biomarkers ( $\mu \mathrm{g} / \mathrm{L})$. & D-Ca (mg) & D-Mg (mg) & $\mathrm{D}-\mathrm{Fe}(\mathrm{mg})$ & $\mathrm{D}-\mathrm{Se}(\mathrm{mcg})$ & D-Cu (mg) & $\mathrm{D}-\mathrm{Zn}(\mathrm{mg})$ \\
\hline B-Ca & 0.15 & 0.17 & 0.06 & 0.11 & -0.04 & -0.002 \\
\hline B-Mg & 0.11 & $0.27 * \star$ & 0.04 & 0.09 & -0.03 & 0.002 \\
\hline $\mathrm{B}-\mathrm{Fe}$ & 0.17 & $0.17 *$ & 0.10 & 0.10 & 0.001 & 0.06 \\
\hline $\mathrm{B}-\mathrm{Cu}$ & -0.02 & 0.17 & 0.04 & 0.02 & -0.04 & 0.001 \\
\hline$B-Z n$ & $0.29 * *$ & $0.18^{*}$ & 0.11 & 0.13 & -0.03 & 0.08 \\
\hline B-Se & 0.16 & $0.21 *$ & 0.07 & 0.07 & -0.06 & 0.002 \\
\hline U-Ca & 0.04 & -0.09 & -0.06 & 0.07 & 0.21 ** & -0.10 \\
\hline U-Mg & -0.003 & -0.10 & -0.07 & 0.03 & -0.06 & -0.10 \\
\hline U-Fe & 0.11 & -0.04 & 0.07 & 0.01 & -0.02 & 0.09 \\
\hline $\mathrm{U}-\mathrm{Cu}$ & -0.05 & $-0.21^{\star *}$ & -0.09 & 0.05 & 0.09 & -0.11 \\
\hline U-Zn & 0.07 & 0.09 & 0.02 & 0.12 & -0.04 & -0.01 \\
\hline U-Se & 0.06 & -0.11 & -0.12 & 0.12 & 0.01 & -0.09 \\
\hline
\end{tabular}

Abbreviations: D-Dietary; U-Urinary; B-Blood

p-value notation: ${ }^{*} p<0.05, * * p<0.01$

\section{Association of sociodemographic characteristics and other work-related factors with dietary micronutrient intake among e-waste recyclers}

We further evaluated micronutrient-intake associations with daily work duration, age, daily income earned, educational status, exposure to biomass burning, cigarette smoking, alcohol intake, BMI and recycler job task (Supplementary Table 4a). Factors such as hours spent recycling e-waste and daily income earned were associated with dietary $\mathrm{Ca}$ and Fe consumption. BMI was also positively associated with the consumption of foods rich in $\mathrm{Mg}, \mathrm{Cu}$ and $\mathrm{Se}$. On the other hand, educational status was inversely associated with dietary $\mathrm{Mg}$ intake ( $\beta=-0.19 ; 95 \% \mathrm{Cl}:-0.36,-0.02, p=0.03)$. Most factors, such as age, job task undertaken, cigarette smoking, exposure to biomass burning and alcohol intake, were not associated with the dietary intake of micronutrient-rich diets.

\section{Relationships between selected work-related factors and blood and urinary micronutrient levels}

Exposure to biomass burning was associated with the lower levels of $\mathrm{Mg}, \mathrm{Zn}$ and Fe measured in the blood of e-waste recyclers (Supplementary Table 4b). Moreover, increased duration of work at the recycling site was inversely proportional to Mg levels in the blood of e-waste recyclers. The nature of e-waste job-task performed significantly influenced blood levels of Ca, Mg, Zn and $\mathrm{Cu}$, with collectors/ sorters having the highest. However, factors such as daily work duration, age, BMI, cigarette smoking and alcohol intake did not influence the blood micronutrient levels. 
We also investigated the associations between job-related factors with urinary micronutrient excretion (Supplementary Table 4c). Significant relationships were found between years of recycling e-waste and urinary levels of $\mathrm{Ca}(\beta=0.08 ; 95 \% \mathrm{Cl}:-0.01,0.15$, $p=0.02)$ and $\mathrm{Cu}(\beta=0.09 ; 95 \% \mathrm{Cl}: 0.04,0.13, p=0.004)$. Furthermore, we found that cigarette smoking was associated with lower levels of $\mathrm{Ca}, \mathrm{Mg}$, and $\mathrm{Zn}$ excreted in urine of e-waste recyclers. Urinary levels of $\mathrm{Cu}$ were also lower with increasing age ( $\beta$ $=-0.07 ; 95 \% \mathrm{Cl}:-0.11,0.03, p=0.004$ ). Meanwhile, exposure to biomass burning as well as factors such as alcohol intake, $\mathrm{BMI}$, job task undertaken and the number of hours (per day) spent recycling e-waste did not have any significant influence on urinary levels of the micronutrients measured.

\section{Discussion}

Recycling of electronic waste (e-waste) is growing worldwide (Heacock et al., 2016; Heacock et al., 2018). It is largely an informal sector activity situated in low- and middle-income countries thus drawing from populations whose dietary intake do not have the full complement of required micronutrients (Basu et al., 2016). Despite the growing concerns over health risks in these communities, no study to our knowledge has assessed the nutritional status of e-waste recyclers. Such an assessment is needed to increase understanding of the health of e-waste recyclers, and to better interpret environmentally-related health risks due to exposure of e-waste recyclers to toxicants which has been the primary focus of most studies. To address this knowledge gap, our study is one of the first to estimate in detail the micronutrient status of e-waste recyclers, using both dietary survey information and biomarker data. We measured levels of micronutrients (Cu, Fe, Se and Zn) previously assessed by Srigboh et al. (2016) as well as $\mathrm{Ca}$ and $\mathrm{Mg}$ in whole blood and urine of e-waste recyclers at Agbogbloshie, Ghana. Subsequently, the levels of these micronutrients were compared to reference values proposed by lyengar and Woittiez (1988) and Alimonti et al. (2005) due to the absence of a national reference range values for Ghana (or Africa). Overall, among the e-waste recyclers, the blood levels of Ca, Se and $\mathrm{Zn}$ reported here were within the proposed reference ranges. Meanwhile, among the controls, all the micronutrients measured in both the blood and urine fell within the reference ranges. There were differences in micronutrient intake or biomarker levels according to variables noted in our a priori objectives and these are discussed below.

\section{Dietary micronutrient intake (based on food models) of e-waste recyclers and controls}

Generally, dietary intake of $\mathrm{Fe}$ and $\mathrm{Zn}$ was adequate among the e-waste recyclers probably owing to their regular intake of traditional green leafy soups. However, there was a high probability of inadequate dietary intake of $\mathrm{Mg}, \mathrm{Ca}$, Se and Cu-rich diets in both e-waste recyclers and controls when compared to the RDA proposed by WHO. Particularly, the mean levels of $\mathrm{Ca}$, Se and $\mathrm{Mg}$ estimated from the diet were lower among the e-waste recyclers, while the mean level of $\mathrm{Zn}$ intake was lower among the controls. These results are consistent with similar outcomes in Malawi (Joy et al., 2015) and South Africa (Kolahdooz et al., 2013) where $\mathrm{Ca}$ and Se intake were inadequate among adult males (general population). In addition, these findings seem to suggest micronutrient deficiency as a common public health issue among healthy males in sub-Saharan Africa. Studies have revealed micronutrient deficiencies in long-term heat-exposed steel recyclers as possibly due to huge losses through sweat and its further association with reduced appetite (Siquier-Coll et al., 2019; Tang et al., 2016). In our study, burners, who are highly exposed to heat, poorly consumed $\mathrm{Zn}$-rich foods believed to alleviate heat stress. Other studies have also predicted deficiencies in micronutrients (such as $\mathrm{Ca}, \mathrm{Cu}, \mathrm{Mg}$ and $\mathrm{Se}$ ) to be associated with increased exposure to toxic metals and other pollutants released during the informal processing of e-waste (Bharatraj \& Yathapu, 2018; Miller \& Rayalam, 2017; Schulz et al., 2015). This suggests that in populations such as the informal sector of e-waste recyclers where toxicant exposures seem to be apparent, a public health strategy targeted at adequately increasing dietary intake of micronutrient-rich diets is critical.

\section{Micronutrient levels in blood and urine of ewaste recyclers and controls}

The analytical quality of the blood and urine measurements was good, and even though we did not have a reference material to gauge the accuracy of the $\mathrm{Ca}, \mathrm{Fe}$ and $\mathrm{Mg}$ measures we do note that the values found here fall within normal reference ranges thus giving us some confidence over their validity. Blood levels of $\mathrm{Ca}, \mathrm{Mg}$, Se and Fe were significantly lower among the e-waste recyclers than the controls, with mean $\mathrm{Mg}$ and Fe levels of the e-waste recyclers falling below the reference ranges. In comparison with Srigboh et al. (2016) who previously conducted a study in the same site, our current study detected higher blood levels of Cu and Zn among e-waste recyclers, but lower blood levels of Fe and Se. Furthermore, the blood level of Fe of e-waste recyclers was below the reference range used. Blood Fe levels of e-waste recyclers may not reflect the overall Fe status, as judging only by the 
low blood Fe levels indicate Fe deficiency, especially when their Fe dietary intake met the RDA. This hypothesis may need to be supported by relevant biomarkers (i.e., hemoglobin, total iron-binding capacity, transferrin receptor and ferritin), which were not analyzed in this study. Poor blood $\mathrm{Ca}$ and $\mathrm{Mg}$ levels of the e-waste recyclers may be attributed to a higher intake of micronutrients such as $\mathrm{Cu}$ and Fe from their diet, which is believed to inhibit the absorption of $\mathrm{Ca}$ and $\mathrm{Mg}$. For instance, at the e-waste scrapyard, recyclers have a long-standing habit of consuming a lot of tea and other herbs believed to contain $\mathrm{Cu}, \mathrm{Zn}$ and Fe as well as organic acid, which are known to inhibit the absorption of Ca and Mg (Karak et al., 2017; Sembratowicz \& Rusinek-Prystupa, 2014). In addition, the lower blood levels of $\mathrm{Ca}, \mathrm{Mg}$, Se and Fe among the e-waste recyclers may be related to their varied dietary habits and lower income status compared to the control population. Furthermore, among e-waste recycler groups, the lowest blood Ca levels were detected among the burners, who are highly exposed to heat, which mostly predisposes them to dehydration. In a bid to hydrate themselves, tend to drink a lot of carbonated drinks (mostly vended onsite) that contain phosphoric acid, believed to inhibit Ca and Mg absorption (Guarnotta et al., 2017; Ogur et al., 2007).

The estimation of 24-h mineral excretion is known to give a more representative overview of micronutrient status. We, therefore, found that median urinary levels of all the micronutrients analyzed fell within the reference ranges, indicating adequate micronutrient retention. When we further compared the micronutrient urinary levels detected in this study to the findings of Srigboh et al. (2016), our urinary $\mathrm{Zn}$ and $\mathrm{Cu}$ levels among the e-waste recyclers were higher, implying a growing micronutrient excretion rate over time. Also, we found higher urinary levels of $\mathrm{Mg}, \mathrm{Zn}, \mathrm{Cu}$ and Fe among the e-waste recyclers than the controls. Specifically, between the recycler groups, the amount of $\mathrm{Zn}$ excreted was highest among the collectors/ sorters. The high $\mathrm{Cu}$ levels excreted among burners are possible as one of the key tasks they undertake is to burn plastic off wires to recover $\mathrm{Cu}$ (Srigboh et al., 2016). These higher excretion rates of Mg, Zn, Cu and Fe among the e-waste recyclers may also be attributable to their higher exposure to toxic metals that are believed to interact, displace and remove micronutrients from the body (Goyer, 1995, 1997; Peraza et al., 1998).

\section{Relationship between dietary micronutrient intake and micronutrient levels in the blood and urine of the e-waste recyclers}

The correlations between dietary $\mathrm{Ca}, \mathrm{Mg}, \mathrm{Fe}, \mathrm{Cu}, \mathrm{Se}$, and $\mathrm{Zn}$ and the levels of these micronutrients in whole blood and urine of ewaste recyclers were computed. Contrary to our findings, Hunt and Beiseigel (2009) found no relationship between dietary Ca intake and Zn levels in the blood. However, Wood and Zheng (1997) found an inverse relationship between dietary Ca intake and blood Zn levels. Based on the above contradictory findings, the relationships between these micronutrients in different body fluids remains unclear. We further found an inverse correlation between dietary $\mathrm{Mg}$ intake and urinary Cu levels among the e-waste recyclers. This finding supports the results of Bulat et al. (2012), who reported that Mg supplementation had profound effect on $\mathrm{Cu}$ status in cadmium-exposed animals. We also found a direct relationship between $\mathrm{Mg}$ intake from diet and Fe levels in the blood. Although some studies have attributed Fe deficiency anaemia to excessive intake of Mg-rich diets (Sugimoto \& Yamada, 2019), other studies report otherwise (Cinar et al., 2007; Kimura \& Yokoi, 1996). These descriptive correlation data may form the basis for further studies, and also support the notion of examining multiple elements in studies given that recyclers are exposed to complex mixtures.

\section{The relationship of selected work-related factors and sociodemographic characteristics with dietary micronutrient intake and micronutrient levels in blood and urine of ewaste recyclers.}

Our results suggest that the e-waste recyclers' $\mathrm{Ca}$ and Fe intake from their diet improved significantly with increasing daily income earned as well as number of hours spent recycling e-waste. This may be attributed to the fact that $\mathrm{Ca}$ and Fe containing foods are relatively expensive in urban areas (Borquaye et al., 2017), thus people with higher income status may afford such foods. Similarly, a systematic review confirmed that people who earned higher incomes were more likely to consume Ca and Ferich foods such as lean meats, whole grains, fish and low-fat dairy products (García-Chávez et al., 2018; Mayen et al., 2014). Furthermore, an increase in BMI was associated with an increase in $\mathrm{Mg}$, $\mathrm{Cu}$ and Se intake among the e-waste recyclers. In contrast, other studies have identified negative correlations between BMI and dietary intake of Se (Wang et al., 2016) and Mg (Castellanos-Gutiérrez et al., 2018). Meanwhile, some other studies have found no differences between BMI and dietary intake of Mg, Cu, and Se (Antipatis \& Gill, 2001; Gonoodi et al., 2018; Urbano et al., 2002). These contrasts may be explained by the fact that our study did not consider the intake of dietary supplements, therefore, individual micronutrient intake may be underestimated as supplement users are more likely to meet their daily requirements. To a large extent, factors such as e-waste

Page 12/19 
job task undertaken, cigarette smoking, exposure to biomass burning and alcohol intake were not associated with the micronutrients measured in this study.

Generally, information about the effect of cigarette smoking on micronutrient status is scarce, except for Se and Cu. Our study found no significant relationship between cigarette smoking and micronutrient levels in blood. However, results from past studies have revealed that there is a significantly lower level of micronutrients in blood of cigarette smokers (Afridi et al., 2015; Jenifer et al., 2015; Messner \& Bernhard, 2014). In addition, other studies have also found an inverse relationship between serum Zn and Cu levels and cigarette smoking; however, these relationships were left unexplained (Ghayour-Mobarhan et al., 2005; Kim et al., 2002; Tungtrongchitr et al., 2003). Drawing parallels from these findings, the effects of cigarette smoking on micronutrient status may be dependent on the type, quantity as well as how smoking is done. Our study did not consider all these factors, thus the possible lack of relationship observed. Moreover, we found that exposure to biomass burning significantly reduced blood levels of Fe, Mg and $\mathrm{Zn}$ among the e-waste recyclers. This negative association between exposure to biomass burning and blood Fe levels, for instance, may probably be the reason for the low blood Fe levels measured among e-waste recyclers, which may consequently serve as a risk factor for anaemia. Furthermore, we observed that prolonged stay and recycling of e-waste over time may be associated with reduction in $\mathrm{Mg}$ absorption in the blood of e-waste recyclers. These lower blood $\mathrm{Mg}$ levels may further be linked to increased exposure to toxicants such as $\mathrm{Pb}$, As and $\mathrm{Cd}$, which are released during informal e-waste recycling activities. The differences in blood levels of $\mathrm{Ca}, \mathrm{Mg}, \mathrm{Zn}$, and Cu found among the e-waste recycler groups may be attributed to their job task and degree of exposure to toxicant as evident in Srigboh et al. (2016) findings.

Assessing the association between work-related factors, sociodemographic characteristics and urinary excretion of micronutrients, we found that prolong years of e-waste recycling directly influenced the amount of $\mathrm{Ca}$ and $\mathrm{Cu}$ excreted. Toxic metals such as $\mathrm{Cd}$ and $\mathrm{Pb}$ have slower excretion rates from the body, yet long half-lives (e.g., the half-life of $\mathrm{Pb}$ is 27 years in cortical bone and 16 years in cancellous bone, whereas Cd has a half-life of 10-30 years) (Alissa \& Ferns, 2011). In view of these, working at the e-waste site for at least 10 years may pre-empt already bioaccumulated metals to interact, displace and excrete micronutrients such as $\mathrm{Ca}$ and $\mathrm{Cu}$ through the urine in humans. In addition, we found that as age increases, the amount of $\mathrm{Cu}$ excreted in urine reduced among the e-waste recyclers.

\section{Limitations And Strengths}

This study has several strengths. It is well known that dietary intake surveys are mostly challenged by the risk of under-and/or over-reporting. Our study therefore employed biomarker analysis of micronutrients in biological samples (blood and urine) to help support the survey results. This approach may provide a more accurate and holistic understanding of micronutrient status. This study is arguably the largest examination of micronutrient status among e-waste recyclers, and is situated in one of the best studied e-waste sites worldwide and as such benefits from a range of other data and knowledge available. However, in future studies, the use of repeated measurements of micronutrient biomarkers will be required to better characterise the micronutrient status of the recyclers and their effects on health outcomes. Further, we asked questions about job activities and present a simple interpretation here (3 general categories) though other studies have shown that e-waste recyclers tend to perform many tasks onsite (Laskaris et al., 2019). We tried to put the findings into context by comparing values with "background populations" but there is a lack of understanding of such values from Africa, especially from vulnerable recycler populations based in low- and middleincome countries.

\section{Conclusion}

In conclusion, our findings highlight inadequate intake of $\mathrm{Ca}, \mathrm{Mg}$, and $\mathrm{Cu}$ rich-diets among e-waste recyclers and also controls. Clearly, a coordinated multi-micronutrient program is needed to combat the co-existing micronutrient deficiencies in not only ewaste recyclers but also the general population; at least in those with poor income status in the region based on a review of the data from the controls. We also found that median urinary levels of all the micronutrients analyzed fell within reference ranges as established by lyengar and \& Woittiez in 1988. Although not conclusive, the lower blood levels of Fe among e-waste recyclers may indicate Fe deficiency. We further identified that exposure to biomass burning was associated with lower blood levels of Fe, Mg and $\mathrm{Zn}$ among the e-waste recyclers, which may consequently serve as a risk for developing anaemia. Prolong stay and recycling

Page 13/19 
of e-waste coupled with exposure to toxic metals that have a half-life as long as 10 years may increase the risk of micronutrient excretion among e-waste recyclers than controls. This may serve as a risk of micronutrient deficiency among e-waste recyclers. Furthermore, the inadequate intake of Zn-rich foods among the burners, coupled with their increased risk of heat exposure, negatively influences their $\mathrm{Zn}$ status.

Specific RDAs, as well as biomarker reference levels tailored to toxicant-exposed groups, remain absent, making the comparisons unparalleled. We, therefore, recommend the need to obtain micronutrient biomarker reference levels for toxicant-exposed groups, such as the e-waste recyclers. Furthermore, country-specific reference levels for biomarkers need to be set for ideal comparisons. In addition, studies should be conducted to identify appropriate RDAs for toxicant-exposed groups, as they may have a higher requirement than the allowances for healthy people. We also recommend nutrition program interventions, including micronutrient supplementation, education and health monitoring for e-waste recyclers who are believed to be at risk of malnutrition. Beyond that, tailored nutrition-related dialogues are required to educate informal e-waste recyclers on the ideal intake of specific nutrients of concern and their impact on nutritional status in order to improve the health of people in a highly polluted environment.

\section{List Of Abbreviations}

E-waste: Electronic waste

Ca: Calcium

Fe: Iron

Se: Selenium

Mg: Magnesium

Zn: Zinc

Cu: Copper

B-Ca: Blood Calcium

B-Cu: Blood Copper

B-Zn: Blood Zinc

B-Mg: Blood Magnesium

B-Se: Blood Selenium

B-Fe: Blood Iron

U-Ca: Urinary Calcium

U-Cu: Urinary Copper

U-Zn: Urinary Zinc

U-Mg: Urinary Magnesium

U-Se: Urinary Selenium

U-Fe: Urinary Iron

RDA: Recommended Daily Allowance 
WHO: World Health Organization

UNFPA: United Nations Population Fund

\section{Declarations}

\section{Ethics approval and consent to participate}

Institutional Review Boards at the University of Ghana, the University of Michigan and McGill University approved the study protocols. The local chief of Agbogbloshie and Madina-Zongo permitted and allowed our research team to enter the community to conduct this study. Written informed consent was sought from each subject before participating in this study.

\section{Consent for publication}

Not applicable

\section{Availability of supporting data}

\section{Competing Interests}

The authors declare that they have no competing interests.

\section{Funding}

This study was financed by the West Africa-Michigan CHARTER in GEO-Health with funding from the United States National Institutes of Health/Fogarty International Center (US NIH/FIC) (paired grant no 1U2RTW010110-01/5U01TW010101) and Canada's International Development Research Center (IDRC) (grant no. 108121-001). Except for providing financial support, the funders played no role in the design of the study, collection, analysis, and interpretation of data and in writing the manuscript.

\section{Authors' contributions}

SAT conceptualized the paper, partook in the investigation process, wrote the methodology, conducted the formal analysis, wrote the original draft, reviewed and edited the paper

NB wrote, reviewed, edited and supervised the writing of the paper

JAM wrote, reviewed, edited and supervised the writing of the paper

DD formally analyzed the data as well as helped in the writing, reviewing and editing of the paper

AAN wrote, reviewed and edited the paper

LK wrote, reviewed and edited the paper

AAA wrote, reviewed and edited the paper

TGR provided resources, reviewed, edited and supervised the writing of the manuscript and also helped in acquiring the funding JNF conceptualized the paper, provided resources, helped in the writing, reviewing, editing, overall supervision as well as the acquisition of funds.

All authors have read and approved the manuscript.

\section{Acknowledgements:}

We further acknowledge the dedicated help of the phlebotomist, trained interpreters and dietitians who facilitated the data collection process. In addition to this, technical assistance in the lab was provided by Andrea Santa-Rios, Hélène Lalande, Tianai 
Zhou, and Jenny Eng.

\section{Authors' information:}

Not Applicable

\section{References}

1. Afridi, H. I., et al. (2015). Estimation of toxic elements in the samples of different cigarettes and their effect on the essential elemental status in the biological samples of Irish smoker rheumatoid arthritis consumers. Environmental monitoring and assessment, 187(4), 157.

2. Alimonti, et al. (2005). Assessment of reference values for selected elements in a healthy urban population. Ann Ist Super Sanita, 41(2), 181-187.

3. Alissa, E. M., \& Ferns, G. A. (2011). Heavy metal poisoning and cardiovascular disease. Journal of toxicology, 2011.

4. Alkhajah, T. A., et al. (2012). Sit-stand workstations: a pilot intervention to reduce office sitting time. American journal of preventive medicine, 43(3), 298-303.

5. Amoabeng Nti, A. A., et al. (2020). Effect of Particulate Matter Exposure on Respiratory Health of e-Waste Workers at Agbogbloshie, Accra, Ghana. International journal of environmental research and public health, 17(9), 3042.

6. Antipatis, V. J., \& Gill, T. P. (2001). Obesity as a global problem. International textbook of obesity, 3-22.

7. Asampong, E., et al. (2015). Health seeking behaviours among electronic waste workers in Ghana. BMC public health, 15(1), 1065.

8. Basu, N., et al. (2016). Occupational and environmental health risks associated with informal sector activities-Selected case studies from West Africa. NEW SOLUTIONS: A Journal of Environmental and Occupational Health Policy, 26(2), $253-270$.

9. Basu, N., et al. (2011). Multiple metals exposure in a small-scale artisanal gold mining community. Environmental research, $117(3), 463-467$.

10. Bharatraj, D. K., \& Yathapu, S. R. (2018). Nutrition-pollution interaction: An emerging research area. The Indian journal of medical research, 148(6), 697.

11. Boateng, G. P. (2014). The development of a photographic food atlas with portion sizes of commonly consumed carbohydrate foods in Accra, Ghana. University of Ghana,

12. Borquaye, L. S., et al. (2017). Nutritional and anti-nutrient profiles of some Ghanaian spices. Cogent Food \& Agriculture, 3(1), 1348185.

13. Bulat, et al. (2012). Effect of magnesium supplementation on the distribution patterns of zinc, copper, and magnesium in rabbits exposed to prolonged cadmium intoxication. The Scientific World Journal, 2012.

14. Cao, H., et al. (2019). Pollution Characteristics and Occupational Exposure Risk of Heavy Metals in Indoor and Outdoor Ambient Particles at a Scaled Electronic Waste Dismantling Plant, Northwest China. Huan jing ke xue= Huanjing kexue, 40(3), 1101-1110.

15. Castellanos-Gutiérrez, A., et al. (2018). Higher dietary magnesium intake is associated with lower body mass index, waist circumference and serum glucose in Mexican adults. Nutrition journal, 17(1), 114.

16. Cinar, V., et al. (2007). Effects of magnesium supplementation on blood parameters of athletes at rest and after exercise. Biological trace element research, 115(3), 205-212.

17. Erickson, A. C., \& Arbour, L. (2014). The shared pathoetiological effects of particulate air pollution and the social environment on fetal-placental development. Journal of environmental and public health, 2014.

18. FAO. (2018). A resource guide to method selection and application in low resource settings. FAO: Rome, Italy.

19. Feldt, T., et al. (2014). High levels of PAH-metabolites in urine of e-waste recycling workers from Agbogbloshie, Ghana. Sci Total Environ, 466-467, 369-376. doi:10.1016/j.scitotenv.2013.06.097

20. García-Chávez, C. G., et al. (2018). Sociodemographic factors are associated with dietary patterns in Mexican schoolchildren. Public health nutrition, 21(4), 702-710. 
21. Ghayour-Mobarhan, M., et al. (2005). Determinants of serum copper, zinc and selenium in healthy subjects. Annals of clinical biochemistry, 42(5), 364-375.

22. Gonoodi, K., et al. (2018). Serum and dietary zinc and copper in Iranian girls. Clinical biochemistry, 54, 25-31.

23. Goyer, R. A. (1995). Nutrition and metal toxicity. The American journal of clinical nutrition, 61(3), 646S-650S.

24. Goyer, R. A. (1997). Toxic and essential metal interactions. Annual review of nutrition, 17(1), 37-50.

25. Guarnotta, V., et al. (2017). The daily consumption of cola can determine hypocalcemia: a case report of postsurgical hypoparathyroidism-related hypocalcemia refractory to supplemental therapy with high doses of oral calcium. Frontiers in endocrinology, $8,7$.

26. Hansen, A. W., et al. (2011). Dietary patterns, food and macronutrient intakes among adults in three ethnic groups in rural Kenya. Public health nutrition, 14(9), 1671-1679.

27. Heacock, M., et al. (2016). E-waste and harm to vulnerable populations: a growing global problem. Environmental Health Perspectives, 124(5), 550-555.

28. Heacock, M., et al. (2018). Prevention-intervention strategies to reduce exposure to e-waste. Reviews on environmental health, 33(2), 219-228.

29. Hennig, B., et al. (2007). Using nutrition for intervention and prevention against environmental chemical toxicity and associated diseases. Environmental Health Perspectives, 115(4), 493-495.

30. Hunt, J. R., \& Beiseigel, J. M. (2009). Dietary calcium does not exacerbate phytate inhibition of zinc absorption by women from conventional diets. The American journal of clinical nutrition, 89(3), 839-843.

31. lyengar, V., \& Woittiez, J. (1988). Trace elements in human clinical specimens: evaluation of literature data to identify reference values. Clinical chemistry, 34(3), 474-481.

32. Jan, A. T., et al. (2015). Heavy metals and human health: mechanistic insight into toxicity and counter defense system of antioxidants. International journal of molecular sciences, 16(12), 29592-29630.

33. Jenifer, H. D., et al. (2015). The influence of cigarette smoking on blood and salivary super oxide dismutase enzyme levels among smokers and nonsmokers-A cross sectional study. Journal of traditional and complementary medicine, 5(2), 100105.

34. Joy, E. J., et al. (2015). Dietary mineral supplies in Malawi: spatial and socioeconomic assessment. BMC Nutrition, 1(1), 42.

35. Kannan, S., et al. (2006). Exposures to airborne particulate matter and adverse perinatal outcomes: a biologically plausible mechanistic framework for exploring potential effect modification by nutrition. Environmental Health Perspectives, 114(11), 1636-1642.

36. Karak, T., et al. (2017). Comparative assessment of copper, iron, and zinc contents in selected indian (Assam) and south African (thohoyandou) tea (camellia sinensis L.) samples and their infusion: A quest for health risks to consumer. Biological trace element research, 175(2), 475-487.

37. Kim, C.-H., et al. (2002). Elevated serum ceruloplasmin levels in subjects with metabolic syndrome: a population-based study. Metabolism-Clinical and Experimental, 51(7), 838-842.

38. Kimura, M., \& Yokoi, K. (1996). Iron accumulation in tissues of magnesium-deficient rats with dietary iron overload. Biological trace element research, 51(2), 177-197.

39. Kolahdooz, F., et al. (2013). Dietary adequacies among South African adults in rural KwaZulu-Natal. PloS one, 8(6), e67184.

40. Kordas, K., et al. (2017). Iron and zinc supplementation does not impact urinary arsenic excretion in Mexican school children. The Journal of pediatrics, 185, 205-210. e201.

41. Kwarteng, L., et al. (2020). Air Quality Impacts at an E-Waste Site in Ghana using Flexible, Moderate-Cost and QualityAssured Measurements. GeoHealth, e2020GH000247.

42. Landrigan, P. J., et al. (2018). The Lancet Commission on pollution and health. The lancet, 391(10119), 462-512.

43. Laskaris, Z., et al. (2019). Derivation of Time-Activity Data Using Wearable Cameras and Measures of Personal Inhalation Exposure among Workers at an Informal Electronic-Waste Recovery Site in Ghana. Ann Work Expo Health. doi:10.1093/annweh/wxz056

Page $17 / 19$ 
44. Mahan, L. K., \& Raymond, J. L. (2016). Krause's food \& the nutrition care process: Elsevier Health Sciences.

45. Mayen, A.-L., et al. (2014). Socioeconomic determinants of dietary patterns in low-and middle-income countries: a systematic review. The American journal of clinical nutrition, 100(6), 1520-1531.

46. Messner, B., \& Bernhard, D. (2014). Smoking and cardiovascular disease: mechanisms of endothelial dysfunction and early atherogenesis. Arteriosclerosis, thrombosis, and vascular biology, 34(3), 509-515.

47. Miller, C. N., \& Rayalam, S. (2017). The role of micronutrients in the response to ambient air pollutants: Potential mechanisms and suggestions for research design. Journal of Toxicology and Environmental Health, Part B, 20(1), 38-53.

48. Moore, L. L., et al. (2012). Food group intake and micronutrient adequacy in adolescent girls. Nutrients, 4(11), 1692-1708.

49. Nti, A. A. A. (2015). Effects of E-Waste on Respiratory Function Among E-Waste Workers Engaged In Burning At Agbogbloshie, Accra. University Of Ghana,

50. Ogur, R., et al. (2007). Evaluation of the effect of cola drinks on bone mineral density and associated factors. Basic \& clinical pharmacology \& toxicology, 100(5), 334-338.

51. Peraza, M. A., et al. (1998). Effects of micronutrients on metal toxicity. Environmental Health Perspectives, 106(Suppl 1), 203. Retrieved from https://www.ncbi.nlm.nih.gov/pmc/articles/PMC1533267/pdf/envhper00536-0213.pdf

52. Prakash, S., et al. (2010). Socio-economic assessment and feasibility study on sustainable e-waste management in Ghana. Öko-Institut eV in cooperation with Ghana Environmental Protection Agency (EPA) \& Green Advocacy Ghana, Ministry of Housing, Spatial Planning and the Environment, VROM-Inspectorate.

53. Risk Factors Collaborators. (2016). GBD (2017). Global, regional, and national comparative risk assessment of 84 behavioural, environmental and occupational, and metabolic risks or clusters of risks, 1990-2016: A systematic analysis for the Global Burden of Disease Study 2016. Lancet, 390(10100), 1345-1422.

54. Schulz, A. J., et al. (2015). Effects of particulate matter and antioxidant dietary intake on blood pressure. American journal of public health, 105(6), 1254-1261.

55. Schwalfenberg, G. K., \& Genuis, S. J. (2015). Vitamin D, essential minerals, and toxic elements: exploring interactions between nutrients and toxicants in clinical medicine. The Scientific World Journal, 2015.

56. Sembratowicz, I., \& Rusinek-Prystupa, E. (2014). Effects of Brewing Time on the Content of Minerals in Infusions of Medicinal Herbs. Polish Journal of Environmental Studies, 23(1).

57. Simon, S. (2018). From Europe, to the Agbogbloshie Scrapyard. In.

58. Singh, M., et al. (2018). Health risk assessment of the workers exposed to the heavy metals in e-waste recycling sites of Chandigarh and Ludhiana, Punjab, India. Chemosphere, 203, 426-433.

59. Siquier-Coll, J., et al. (2019). Effect of heat exposure and physical exercise until exhaustion in normothermic and hyperthermic conditions on serum, sweat and urinary concentrations of magnesium and phosphorus. Journal of thermal biology, 84, 176-184.

60. Srigboh, R. K., et al. (2016). Multiple elemental exposures amongst workers at the Agbogbloshie electronic waste (e-waste) site in Ghana. Chemosphere, 164, 68-74.

61. Sugimoto, H., \& Yamada, U. (2019). Iron deficiency anemia induced by magnesium overuse: a case report. BioPsychoSocial Medicine, 13(1), 18.

62. Takyi, S. A., et al. (2020). Micronutrient-rich dietary intake is associated with a reduction in the effects of particulate matter on blood pressure among electronic waste recyclers at Agbogbloshie, Ghana.

63. Tang, Y.-M., et al. (2016). Relationships between micronutrient losses in sweat and blood pressure among heat-exposed steelworkers. Industrial health, 2014-0225.

64. Tchounwou, P. B., et al. (2012). Heavy metal toxicity and the environment. In Molecular, clinical and environmental toxicology (pp. 133-164): Springer.

65. Tungtrongchitr, R., et al. (2003). Serum copper, zinc, ceruloplasmin and superoxide dismutase in Thai overweight and obese. Journal of the Medical Association of Thailand= Chotmaihet thangphaet, 86(6), 543-551. 
66. United Nations Population Fund. (2018). Reaching the Underserved: UNFPA Youth Fellows Organizes Outreach at Old Fadama. doi:https://ghana.unfpa.org/en/news/reaching-underserved-unfpa-youth-fellows-organizes-outreach-old-fadama

67. Urbano, M. R., et al. (2002). Iron, copper and zinc in adolescents during pubertal growth spurt. Jornal de pediatria, 78(4), 327334.

68. Wang, Y., et al. (2016). Significant beneficial association of high dietary selenium intake with reduced body fat in the CODING Study. Nutrients, 8(1), 24.

69. Wittsiepe, J., et al. (2017). Pilot study on the internal exposure to heavy metals of informal-level electronic waste workers in Agbogbloshie, Accra, Ghana. Environmental Science and Pollution Research, 24(3), 3097-3107.

70. Wood, R. J., \& Zheng, J. J. (1997). High dietary calcium intakes reduce zinc absorption and balance in humans. The American journal of clinical nutrition, 65(6), 1803-1809.

71. Zeba, A. N., et al. (2014). Dietary patterns and physical inactivity, two contributing factors to the double burden of malnutrition among adults in Burkina Faso, West Africa. Journal of nutritional science, 3.

\section{Supplementary Files}

This is a list of supplementary files associated with this preprint. Click to download.

- APPENDIX.docx 